\title{
PROGRAMA DE TÉCNICAS ASERTIVAS PARA MEJORAR LAS RELACIONES INTERPERSONALES DE LOS ESTUDIANTES DE EDUCACIÓN PRIMARIA
}

\author{
PROGRAM ASSERTIVE TECHNIQUES TO IMPROVE RELATIONS \\ INTERPERSONAL STUDENTS OF PRIMARY EDUCATION
}

\section{Laura Yrene Guillermo Tantarico ${ }^{1}$}

\section{RESUMEN}

La investigación buscó demostrar que un programa de técnicas asertivas mejora las relaciones interpersonales de niños y niñas del 5ํ grado de las Instituciones Educativas "Paz y Amistad" y "Augusto Salazar Bondy", de Nuevo Chimbote-2011. La investigación tuvo un diseño cuasi experimental de dos grupos no equivalentes con pre y post test, con una muestra de 120 estudiantes y se aplicó como instrumento de medición un cuestionario. Se determinó que el pre test indica que ambos grupos presentan relaciones interpersonales en el nivel inadecuado, situación que varía al aplicar el post test donde los estudiantes del grupo experimental, en un $96,7 \%$, se ubica en el nivel adecuado y solo un 3,3\% en el nivel regular, a diferencia del grupo control que en un $90 \%$ permanece en el nivel inadecuado y un $10 \%$ en el nivel regular. Se aprecia que las técnicas asertivas contribuyen a la mejora de las relaciones interpersonales en los estudiantes de educación primaria.

PALABRAS ClAVE: Técnicas asertivas, habilidades sociales, relaciones interpersonales.

1 Magister en Educación por la Universidad Nacional del Santa. Directora de la Institución Educativa Simón Bolívar, №88041. lauriprin_14@hotmail.com 


\begin{abstract}
The research aims to show that a program of assertive techniques improve interpersonal relationships of children in the 5th grade of Educational Institutions "Peace and Friendship" and "Augusto Salazar Bondy" Nuevo Chimbote-2011. The research was a quasi-experimental design of two non-equivalent groups with pre and post test with a sample of 120 students and applied as a measuring tool a questionnaire. It was determined that the pre test indicates that both groups have interpersonal relationships at the wrong level, a situation that varies by applying the post test where students in the experimental group, $96.7 \%$, is at the right level and only 3, 3\% in the regular level, unlike the control group by $90 \%$ remains in the inadequate level and $10 \%$ at the regular level. It is appreciated that assertive techniques contribute to the improvement of interpersonal relationships in elementary school students.
\end{abstract}

KEY WORDS: Assertive techniques, social skills, interpersonal relationships.

\title{
INTRODUCCIÓN
}

A nivel internacional Naranjo (2008) afirma que la conducta y la comunicación asertiva son habilidades sociales que pueden desarrollarse para mejorar la calidad de las relaciones humanas; mientras que Llanos (2006) declara que los efectos de un programa de enseñanza en habilidades sociales mejoran las relaciones sociales de los participantes y amplía su repertorio.

A nivel nacional, Puga (2008) declara que los niños que reciben castigo físico y emocional presentan dificultades para establecer patrones de relación eficaces ya que no respetan la autonomía de los demás. Por su parte Gonzales (2002) declara que la aplicación del programa de habilidades sociales contribuye a disminuir significativamente las conductas sin inhibiciones.

La sociedad y el mundo laboral demandan personas que sean capaces de tomar decisiones, liderar grupos, resolver conflictos, trabajar en equipo... ¿Cómo se puede aprender? La respuesta está en el conocimiento y la utilización desde la infancia de estas habilidades, momento en el que el niño(a) está formando su personalidad y aprendiendo a relacionarse. La escuela es un lugar clave para el desarrollo de las habilidades sociales que permitirán su desarrollo integral como persona.

La Institución Educativa y sobre todo los docentes que trabajan con niños deben incluir con más frecuencia en sus proyectos educativos, diseños curriculares y programaciones de áreas y de clase, las habilidades sociales como parte del aprendizaje que el niño(a) debe desarrollar en el periodo de escolaridad. Existen múltiples maneras de realizarlo; esto depende de nuestras características como 
docentes así como de los niños, los objetivos y finalidades y recursos de la Institución Educativa.

La Institución Educativa se halla ante el reto de incluir contenidos del área socio-afectiva en la programación curricular. Fernández (2001), citando a Trianes y Muñoz (1997), plantea que necesitamos programas diseñados desde una perspectiva educativa para ser desarrollados en el aula por los docentes, impregnando las metodologías del profesor, introduciendo nuevos objetivos en el aprendizaje de las materias escolares y ajustados al contexto escolar. Aquí es donde el «Programa de técnicas asertivas», tiene un rol importante, ya que busca mejorar las relaciones interpersonales de los estudiantes en el aula.

La presente investigación se justifica porque su importancia radica en responder a la necesidad de buscar soluciones a los problemas sobre relaciones interpersonales que generan conflictos y afecta la armoniosa convivencia escolar, así como la formación social del educando.

El programa de técnicas asertivas se concibe como un conjunto de procedimientos orientados a desarrollar habilidades para prevenir conflictos sociales, que permita el desarrollo del alumno no sólo en su aspecto académico, sino también en su aspecto social y afectivo. Con el cual se conseguirá en el futuro personas con un mayor conocimiento de sí mismas y de los demás, respetuosas, capaces de aceptar las contrariedades y de promover soluciones.

La escuela tiene que educar para la vida, lo que supone facilitar a los alumnos los instrumentos necesarios para que éstos sean capaces de tener una buena autoestima, tomar decisiones responsables, relacionarse positiva y asertivamente con los demás, resolver conflictos de forma positiva, etc. Esto permitirá un mejor aprendizaje por parte del alumno y un clima en el que el profesorado podrá desempeñar su función de educador, más relajadamente y con una mayor calidad.

La investigación aporta un programa que contempla fundamentos que respaldan su importancia, un diseño didáctico y un conjunto de procedimientos metodológicos que se ejecutan en las 12 sesiones de aprendizaje experimentados.

Sobre la asertividad, Wolpe (1958), citado en Paula (2000) la define como: "la expresión, adecuada, dirigida hacia otra persona, de cualquier emoción que no sea la respuesta de ansiedad". Mientras que Adler (1977), citado en Ministerio de Educación (1999), declara que la asertividad es la "habilidad de comunicarse y expresar tus pensamientos y emociones con confianza y con máxima capacidad". 
En Ministerio de Educación (2007), encontramos que la asertividad se considera como "la habilidad personal que nos permite expresar sentimientos, opiniones y pensamientos, en el momento oportuno, en la forma más adecuada y sin negar, ni desconsiderar los derechos de los demás".

Los conceptos descritos nos llevan a pensar en lo importante que es la asertividad para la armoniosa convivencia con los demás. En este sentido, la asertividad debe traducirse en una conducta asertiva, que según De Galassi (s/f), citado en Ministerio de Educación (1999), sostiene que las personas asertivas expresan sus sentimientos, preferencias, necesidades u opiniones hacia las demás personas, sin forzarlas, ni menospreciarlas, ni usarlas como medios.

El problema de la investigación fue iEn qué medida la aplicación de la propuesta de un Programa de Técnicas Asertivas mejorará las relaciones interpersonales de los estudiantes del 5음 grado de educación primaria de las Instituciones Educativas "Paz y Amistad" y "Augusto Salazar Bondy", de Nuevo Chimbote 2011? La hipótesis se formuló en los siguientes términos: La aplicación de un programa de técnicas asertivas mejora significativamente las relaciones interpersonales de los estudiantes del 5 - grado de las Instituciones Educativas "Paz y Amistad" y "Augusto Salazar Bondy", de Nuevo Chimbote-2011.

El objetivo del trabajo fue demostrar que la aplicación del programa de técnicas asertivas mejora las relaciones interpersonales de niños y niñas de $5^{\circ}$ grado de las Instituciones Educativas "Paz y Amistad" y "Augusto Salazar Bondy", de Nuevo Chimbote-2011.

\section{MATERIALES Y MÉTODOS}

En el desarrollo de la investigación se utilizó el método experimental. Es de diseño cuasi experimental que, según Sánchez y Reyes (1998) es útil cuando se quiere someter a experimentación una metodología de trabajo como el Programa de Técnicas Asertivas.

Es de enfoque cuantitativo, que según Hernández, Fernández y Baptista (2006, p. 5) "usa la recolección de datos para probar hipótesis, con base a la medición numérica y el análisis estadístico, para establecer patrones de comportamiento y probar teorías".

La población estuvo constituida por 292 estudiantes del 5 to. Grado de educación primaria de las instituciones educativas 88240 "Paz y Amistad" y 88047 
"Augusto Salazar Bondy" del distrito de Nuevo Chimbote; y la muestra por 128 estudiantes.

Como instrumento de medición se aplicó el cuestionario de Evaluación de Relaciones Interpersonales a los estudiantes, debidamente validado por el juicio de expertos, para identificar el nivel de la variable dependiente antes de la experimentación; y para conocer el nivel en que se encontraron las relaciones interpersonales después de la experimentación.

Para el procesamiento y análisis de los resultados se ha utilizado la estadística descriptiva simple y para la prueba de las hipótesis estadísticas de la estadística inferencial mediante la aplicación de la t de student.

\section{RESULTADOS}

Tabla 1

NIVEL DE LAS RELACIONES INTERPERSONALES DEL GRUPO EXPERIMENTAL Y GRUPO DE CONTROL EN EL PRE TEST

\begin{tabular}{lccccc}
\hline \multirow{2}{*}{ Nivel } & Puntaje & \multicolumn{2}{c}{ Grupo control } & \multicolumn{2}{c}{ Grupo experimental } \\
\cline { 3 - 6 } & & Frecuencia & $\%$ & Frecuencia & $\%$ \\
\hline Inadecuado & $46-107$ & 59 & 98,3 & 60 & 100,0 \\
Regular & $108-168$ & 1 & 1,7 & 0 & 0,0 \\
Adecuado & $169-230$ & 0 & 0,0 & 0 & 0,0 \\
\hline & Total & 60 & 100,0 & 60 & 100,0 \\
\hline
\end{tabular}

Fuente: Cuestionario aplicado a 5ำ grado de educación primaria.

En la Tabla 1 se presentan, el nivel de las relaciones interpersonales de los alumnos del grupo control y experimental, antes del programa de técnicas asertivas; dando los resultados siguientes:

Los estudiantes del grupo de control en un 98,3\% mostraron un inadecuado nivel en sus relaciones interpersonales, mientras que el grupo experimental se encuentra en un 100,0\% en el mismo nivel.

Como se observa, tanto los estudiantes del grupo experimental como del grupo control presentan un nivel inadecuado en sus relaciones interpersonales antes de la aplicación del programa de técnicas asertivas. 
Tabla 2

NIVEL DE LAS RELACIONES INTERPERSONALES DEL GRUPO EXPERIMENTAL Y GRUPO DE CONTROL EN EL POST TEST

\begin{tabular}{lccccc}
\hline \multirow{2}{*}{ Nivel } & Puntaje & \multicolumn{2}{c}{ Grupo control } & \multicolumn{2}{c}{ Grupo experimental } \\
\cline { 3 - 6 } & & Frecuencia & $\%$ & Frecuencia & $\%$ \\
\hline \multirow{2}{*}{ Inadecuado } & $46-107$ & 54 & 90,0 & 0 & 0,0 \\
Regular & $108-168$ & 6 & 10,0 & 2 & 3,3 \\
Adecuado & $169-230$ & 0 & 0,0 & 58 & 96,7 \\
\hline & Total & 60 & 100,0 & 60 & 100,0 \\
\hline
\end{tabular}

Fuente: Cuestionario aplicado a 5o grado de educación primaria.

En la Tabla 2 se presentan el desarrollo de las relaciones interpersonales en los estudiantes del grupo control y experimental, después de aplicar el programa de técnicas asertivas; dando los resultados siguientes:

Los estudiantes del grupo experimental mejoraron sus relaciones interpersonales, pues el $96,7 \%$ se ubican en el nivel adecuado a diferencia del grupo de control que presenta un $0 \%$ en este nivel.

Solo un 3,3\% de los estudiantes del grupo experimental presentan el desarrollo de las relaciones interpersonales en el nivel regular y el 10,0\% del grupo de control.

Los estudiantes del grupo de control presentan un 90\% en el desarrollo de las relaciones interpersonales en el nivel inadecuado.

Después de la aplicación del programa de técnicas asertivas, los estudiantes del grupo control, en su mayoría, mostraron un nivel inadecuado en el desarrollo de las relaciones interpersonales; sin embargo, en el grupo experimental, la mayoría de los estudiantes se ubicaron en el nivel adecuado, debido a la aplicación del programa.

En la Tabla 3 se muestra la prueba de homogeneidad de varianzas, donde la prueba de Levene ha dado un valor de 10,379, el cual ha generado un nivel de significancia experimental de 0,002 inferior al nivel de significancia fijado por las investigadoras $(\alpha=0,05)$, esto permite decidir que no existe igualdad de varianzas de manera significativa. Por otro lado en la evaluación cuantitativa sobre el desarrollo de las habilidades de autoafirmación a partir de los indicadores estadís- 
Tabla 3

EFICACIA DEL PROGRAMA DE TÉCNICAS ASERTIVAS EN LA MEJORA

DE LA AUTOAFIRMACIÓN, AL APLICAR EL POST TEST AL GRUPO EXPERIMENTAL Y CONTROL

Prueba de Homogeneidad de varianzas

Prueba de Levene para la igualdad de varianzas

\begin{tabular}{lcc} 
& F & Sig. \\
\hline Se han asumido varianzas iguales & 10,379 & 0,002 \\
No se han asumido varianzas iguales & &
\end{tabular}

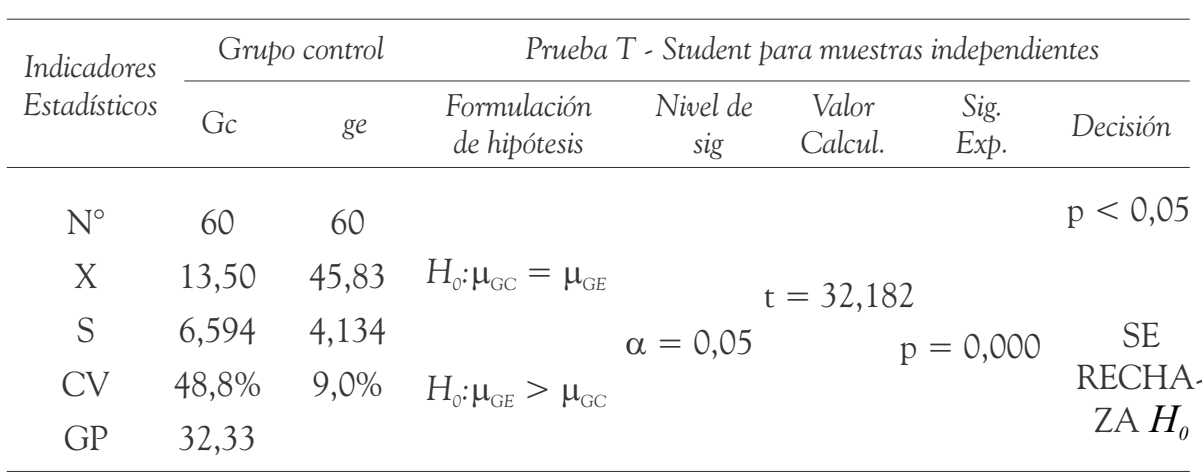

$\mu_{\mathrm{GC}}$ : Calificación media del GC en el Pos test sobre la autoafirmación.

$\mu_{\mathrm{GE}}$ : Calificación media del GE en el Pos test sobre la autoafirmación.

GP: Ganancia Pedagógica.

ticos, se ha logrado un promedio de 13,50 en el grupo control y un promedio de 45,83 en el grupo experimental generando una ganancia pedagógica externa de 32,33 puntos del grupo experimental respecto del grupo control. Así mismo el grupo experimental ha obtenido un coeficiente de variación $(9,0 \%)$ inferior al coeficiente de variación del grupo control (48,8\%); esto manifiesta que las habilidades de autoafirmación en el grupo experimental es más estable respecto del grupo control.

En la Tabla 3 se presentan la prueba de hipótesis para la diferencia de medias en muestras independientes entre el grupo control y grupo experimental. En efecto, esta diferencia fue validada por la Prueba T - Student, al obtener una evidencia suficiente de los datos sobre las habilidades de autoafirmación para generar un nivel de significancia experimental $(p=0,000)$ inferior al nivel de significancia 
fijado por las investigadoras $(\alpha=0,05)$, rechazando la hipótesis nula $H_{0}$ y aceptando la hipótesis alterna $H_{a}$. Esto permite concluir que el programa de técnicas asertivas estrategia influyó de manera muy significativa en el desarrollo de autoafirmación de los estudiantes del grupo experimental respecto del grupo control, con niveles de confianza del $95 \%$.

\section{DISCUSIÓN}

Al examinar los resultados obtenidos, para el objetivo general Demostrar que la aplicación del programa de técnicas asertivas mejora las relaciones interpersonales de niños y niñas de $5^{\circ}$ grado de las instituciones educativas "Paz y Amistad" y "Augusto Salazar Bondy", de Nuevo Chimbote; se puede ubicar en la Tabla 1 que las relaciones interpersonales del grupo de control (GC) antes de la aplicación del programa de técnicas asertivas se presentaba en un 98,3\% en el nivel inadecuado, mientras que el grupo experimental (GE) en un 100,0\% se presentaba en el mismo nivel; esto respalda la problemática detectada y reforzó la necesidad de intervención para mejorar las relaciones interpersonales.

Tal como indica García (1997) "las relaciones se plantean en términos de las distintas posturas que adoptan las personas con respecto a otras próximas", siendo las actitudes de reserva, competitividad, absentismo, intolerancia y frustración que presentan los estudiantes en el aula, factores que estarían afectando sus relaciones interpersonales. Un aspecto afectado es la comunicación, que según Cruz (2002) es muy importante ya que el éxito en la vida depende en gran parte de la manera de relacionarnos con las demás personas, y la base de estas relaciones interpersonales es la comunicación.

Lo expuesto se complementa con lo presentado por la Tabla 2 donde después de aplicar el programa de técnicas asertivas, los integrantes del GE mejoraron sus relaciones interpersonales, pues el $96,7 \%$ se ubican en el nivel adecuado y el porcentaje restante en el nivel regular; a diferencia del grupo de control que presentan un $90 \%$ en el desarrollo de las relaciones interpersonales en el nivel inadecuado. Resultado que presenta semejanza por lo hallado por Naranjo (2008), quien en su investigación refiere que la comunicación asertiva, aspecto trabajado por el Programa de técnicas asertivas, favorece la libertad de ser, la autonomía, la madurez, la integridad del yo y la identidad propia; así también, Llanos (2006) en su tesis indica que su programa desarrollado permite mejorar las relaciones sociales de los participantes y ampliar su repertorio de habilidades sociales. 
Desde la experiencia docente se sume como valioso el aporte de estos programas. De esta manera se respalda la afirmación de Monjas (1994) al señalar que los métodos de entrenamiento en habilidades sociales como la comunicación, permite modificar las conductas de relación interpersonal que el niño/a posee pero que son inadecuadas.

Con relación al primer objetivo específico: Identificar el nivel de las relaciones interpersonales en los estudiantes del $5^{\circ}$ grado de educación primaria, antes de aplicar el programa de técnicas asertivas, considerando las dimensiones de las relaciones interpersonales, encontramos datos respecto a la interacción social; donde el grupo de control presenta un 93,3\% en el nivel inadecuado, mientras que el grupo experimental presenta un 100\% en el mismo nivel; lo cual desde la experiencia docente constituyó un indicador clave para la puesta en marcha del programa; considerando lo señalado en el fundamento teórico de la tesis, que indica que, las conductas y habilidades de interacción social se aprenden, permite entonces, incorporar principios mediante entrenamiento para las personas que todavía no los han adquirido (Nelly, 1987).

Además, respecto a la iniciación de la interacción social y conversacional que el GE presenta un $95 \%$ en el nivel inadecuado, mientras el GC lo hace en un 98,3\%; a pesar de que estos resultados son alarmantes, es importante asumir la indicación de Monjas (1993): ninguna persona nace o desarrolla rápidamente todas sus habilidades sociales... a lo largo de la vida se va aprendiendo las respuestas que emitirá la persona en una situación interpersonal y en su relación con el medio social.

Otros de los resultados que referente a la dimensión cooperar y compartir, indica que el 100\% del GE presentaban un nivel inadecuado, mientras que el GC se ubica en un $96,7 \%$ en este nivel, se refleja en ellos la necesidad del programa; considerando que si una habilidad social no se pone de manifiesto en una situación determinada puede ser debido a un reforzamiento infrecuente, por lo tanto, hay que reforzar positivamente dicha conducta. También respecto a las habilidades de la dimensión emociones, se puede ubicar que el GE y el GC presentan un nivel inadecuado con un 100,0\%; porcentaje que como investigadoras llama la atención profundamente; es decir, si las relaciones en el aula están sentadas sobre la base de actitudes negativas, como la competitividad, la intolerancia y la frustración, el individualismo, entonces las relaciones interpersonales en el aula será negativo. Asimismo, en la dimensión autoafirmación; que el GE ubica un 100\% 
en el nivel inadecuado, mientras el GC registra un 95,0\% en el mismo nivel, confirmando las necesidades señaladas en párrafos anteriores.

En común con los resultados presentados para el objetivo específico se ubica las investigaciones desarrolladas por Puga (2008) quien refiere que cuando los niños viven en ambientes inadecuados presentan de por sí dificultades para relacionarse con su entorno, ya que este no facilita los intercambios positivos, obstruyendo el desarrollo de sentimientos de autoestima en los niños; el mismo autor indica que los niños que recibieron castigo físico y emocional tienen el deseo de vincularse con otros pero, presentan dificultades para establecer relación eficaces ya que no respetan la autonomía de los demás.

Desde el fundamento teórico de la tesis, ubicamos a Trianes y Muñoz (1997), quienes resaltan la necesidad de programas diseñados desde una perspectiva educativa para ser desarrollados en el aula por los docentes, innovando sus prácticas educativas. En la actualidad es prácticamente unánime la opinión de que las habilidades sociales que favorecen las relaciones interpersonales se adquieren a través de interacciones comunicativas significativas.

Sobre los resultados referido al tercer objetivo específico: Identificar el nivel de las relaciones interpersonales los estudiantes del 5 o grado de educación primaria, después de aplicar un programa de técnicas asertivas; la Tabla 3 respecto al desarrollo de las dimensiones de las relaciones interpersonales, encontramos que la interacción social; indican que los estudiantes del GE mejoraron significativamente, pues un $80 \%$ se encuentra en el nivel adecuado, el porcentaje restante en el nivel regular. Por su parte el GC muestra un resaltante $90.0 \%$ en el nivel inadecuado. Encontrando diferencias notorias en el avance del primer grupo. Sobre el particular encontramos a García (1997), quien indica que las relaciones se basan en actitudes positivas como: cooperación, acogida, autonomía, participación, satisfacción; tan necesarias en los sistemas educativos actuales. El investigador Naranjo (2008), concluye en su tesis la importancia de la asertividad como base de la autoafirmación e incluye componentes de tipo ético, como el respeto hacia sí y hacia las otras personas, pues parte del concepto de igualdad de derechos.

Respecto al desarrollo de la iniciación de la interacción social y conversacional; que el GE se ubica en un 93,3\% en el nivel adecuado; mientras que el GC no ha mostrado mayores mejorías indicando un $85,0 \%$ en el nivel inadecuado. Sabemos que los niños y niñas comparten espacios y tiempos de sus vidas y en muchas ocasiones no sólo se relacionan a nivel de estudios, sino que por afinidad logran 
crear vínculos afectivos que muchas veces trascienden el ámbito educativo, estos espacios deberían ser aprovechados por todos los docentes, siguiendo la orientación de Naranjo (2008) que afirma que la conducta y la comunicación asertiva son habilidades sociales que pueden desarrollarse para mejorar la calidad de las relaciones humanas; en el caso de la investigación esta experiencia fue recogida y considerada, canalizando su aprendizaje de las relaciones interpersonales a través de la comunicación.

Otros resultados sobre la habilidad para cooperar y compartir, donde el GE mejoró significativamente con un 83,3\% en el nivel adecuado, por su parte el GC no muestra mejoría ubicando igual porcentaje, pero en el nivel inadecuado, estos resultados de la experiencia de las investigadores resalta la importancia de generar en las instituciones escolares acciones que favorezcan la cooperación a través del trabajo en equipo y el valor de solidaridad que conlleve al compartir, aspectos ejecutados durante las sesiones desarrolladas como parte del programa.

Por otro lado, en el desarrollo de las emociones, que el GE mejoró significativamente pues un 93,3\% se encuentra en el nivel adecuado; mientras que, el GC ubica un mayoritario 90,0\% en el nivel inadecuado.

Sobre la base de los resultados señalados se puede indicar que, si las relaciones se establecen sobre la base de actitudes positivas, como la cooperación, la acogida, la participación, la autonomía y el diálogo, tal como sucedió en el desarrollo del programa, el niño y niña participante logro la valoración positiva de los demás y de sí mismo, así como de la confianza, el clima del aula es positivo y gratificante.

Sobre el particular concordamos con los hallazgos de Naranjo (2008), que en su tesis indica que el comportamiento asertivo le permite a las personas sentirse mejor consigo mismas y en su relación con los demás; por su parte desde el fundamento teórico ubicamos a Macia y Méndez (1986) quienes consideran que la persona; mediante una actuación determinada, siguiendo unas reglas establecidas y específicas como las consideradas dentro del programa, modifica su actuación en función del feedback continuado que recibe del entorno, en su progresión hacia la consecución de sus objetivos.

Desde nuestro rol investigador, las técnicas asertivas tuvieron un efecto positivo gracias a que implicaron la expresión directa de la defensa de los propios derechos y opiniones personales, el autorrefuerzo y el refuerzo a los demás, aspecto reforzado los fundamentos teóricos tal como lo indicado por Marroquín y Villa 
(1995) quien afirma que la comunicación interpersonal es la dimensión a través de la cual nos realizamos como seres humanos, es decir, una persona que no mantiene relaciones interpersonales adecuadas, amenazará su calidad de vida.

En relación al mismo objetivo sobre el desarrollo de las habilidades relacionadas con las emociones. El resultado reafirma la idea fundamentada en el marco teórico, la escuela constituye un lugar muy importante para el niño en el que se tendría que garantizar su correcta evolución, así como favorecer su seguridad afectiva y emocional (Escribá, 1999), es en la escuela y específicamente los docentes, quienes desde la experiencia del investigador deben asumir la responsabilidad de orientar a los estudiantes, como una vía para prevenir e intervenir el maltrato entre iguales, favorecer unas relaciones personales saludables y equilibradas entre ellos y así, mejorar el clima de convivencia escolar.

Asimismo, la Prueba T - Student, indica un nivel de significancia experimental $(\mathrm{p}=0,000)$ inferior al nivel fijado por las investigadoras $(\alpha=0,05)$, rechazando la hipótesis nula $H_{0}$ y aceptando la hipótesis alterna $H_{a}$, tomando en cuenta lo señalado, se citan los resultados similares en la investigación presentada por Gonzales (2002) quien indica que el programa contribuyó a disminuir significativamente las conductas observándose cambios tales como: pedir por favor, dar gracias, expresar quejas, saber conversar e interactuar de manera adecuada con los demás. Asimismo, desde el marco teórico se referencia a Monjas (1993), quien indica que no es más hábil el que más conductas tenga sino el que más capaz sea de percibir y discriminar las señales del contexto y elegir la combinación adecuada de conductas para esa situación determinada.

\section{REFERENCIAS BIBLIOGRÁFICAS}

Escribá, A.; Arnaiz, P.; y Berruezo, P. (1999). Psicomotricidad. Fundamentos teóricos aplicables en la práctica. Madrid: Gymnos.

García R., F. (1997). Organización escolar y gestión de centros educativos. Málaga: Aljibe

Gil Pérez, D. (2001). La innovación en algunos aspectos esenciales -pero habitualmente olvidadosen el planteamiento de la enseñanza/ aprendizaje de las ciencias: las relaciones enseñanzamedio y el clima escolar.

Recuperado el 18 de julio del 2011 en www.oei.org.co/oeivirt/gil02c.htm

Gonzales, M. (2002). "Efectos de la aplicación de un programa de habilidades sociales sobre los problemas de comportamiento de las alumnas del 6to. Grado de primaria del CEP Sagrado Corazón de la ciudad de Trujillo”. Tesis de licenciatura. Universidad César Vallejo. 
Hernández, R., Fernández, C. y Baptista, P. (2006). Metodología de la investigación. 4ta. Edición. México D.F: Mc Graw Hill Interamericana

Llanos, C. (2006). "Efectos de un programa de enseñanza en habilidades sociales". Tesis doctoral. Universidad de Granada.

Marroquín, M. y Villa, A. (1995). La Comunicación Interpersonal. Medición y estrategias para su desarrollo. Bilbao: Mensajero.

Ministerio de Educación. (1999). (2eed.). Guía de educación familiar y sexual. Lima: Impresiones Minedu.

Ministerio de Educación. (2007). (2º ed.). Guía para el desarrollo de la capacidad de toma de decisiones. Lima: Impresiones Minedu.

Monjas, M. (1999). Programa de enseñanza de habilidades de interacción social para niños y niñas en edad escolar (PEHIS). Madrid: CEPE.

Montagu, A. y Matson, F. (1989). El contacto humano. Barcelona: Paidós.

Naranjo, M. (2008). Perspectivas sobre comunicación. Revista electrónica Actualidades Investigativas en Educación. Universidad de Costa Rica.

Recuperado de http://redalyc.uaemex.mx/redalyc/pdf/447/44760101.pdf

Paula, I. (2000). Habilidades Sociales: Educar hacia la autorregularización. Barcelona: Horsori.

Puga, L. (2008). "Relaciones interpersonales en un grupo de niños que reciben castigo físico y emocional”. Tesis de maestría. Pontificia Universidad Católica del Perú.

Sánchez, H. y Reyes, C. (1998). Metodología y Diseño en la Investigación Científica. Lima, Perú: Mantaro

Trianes, M.V. Jiménez, M. y Muñoz, A (1997). Las relaciones interpersonales: Evaluación e intervención. Madrid: Pirámide. 